\title{
A BDF2 Energy-Stable Scheme for a General Tensor- Based Model of Liquid Crystals
}

\author{
Guanghua Ji* \\ Laboratory of Mathematics and Complex Systems, Ministry of \\ Education and School of Mathematical Sciences, Beijing Normal \\ University, Beijing 100875, China.
}

Received 29 October 2018; Accepted (in revised version) 7 June 2019.

\begin{abstract}
Following the scalar auxiliary variable strategy, a linear semi-discrete scheme in time for the hydrodynamic Q-tensor model of liquid crystal polymers is developed. It is shown that the scheme is unconditionally energy stable and uniquely solvable. Numerical simulations show the decreasing energy and the second-order convergence.
\end{abstract}

AMS subject classifications: 49Mxx, 65M12, $81 \mathrm{~T} 80$

Key words: Q-tensor model, unconditional energy stability, linear second order BDF2 scheme.

\section{Introduction}

Liquid crystals represent an intermediate state of matter between crystalline solids and isotropic fluids. Nematic liquid crystals usually have molecular orientational order but not a positional order. The most popular mathematical model for the flow of low molecular weight nematic liquid crystals is the Ericksen-Leslie model [10], where the orientation of molecules is expressed by a unit vector $\mathbf{d} \in \mathscr{S}^{2}$. The distortional elasticity is described by the Oseen-Frank energy but in this case, only uniaxial liquid crystals can be modeled. If the orientational symmetry is broken, the Ericksen-Leslie theory fails to capture the asymmetrical feature of the system. Moreover, if defects emerge, the director model is singular and $\mathbf{d}$ cannot be determined. In order to model liquid crystal droplets, Diegel et al. [3] coupled the Ericksen's model for nematic liquid crystals and the Cahn-Hilliard interfacial energy equation.

An alternative method to describe the orientation of nematic liquid crystal systems consists in using a $Q$-tensor - i.e. a second-order tensor of trace zero. The reflective symmetry of the system and biaxiality are naturally built-in into tensor-based theories and defects can also be captured. Furthermore, the director model can be derived from Q-tensor theory for weak flows and weak elastic limits [20]. Therefore, the Q-tensor based hydrodynamic model is commonly used in nematic liquid crystal flows $[1,5,6,18-20]$.

*Corresponding author. Email address: ghji@bnu.edu.cn (G. Ji) 
In this work, we describe the average orientation of nematic liquid crystals with $\mathbf{Q}$. Set

$$
\Lambda:=\left\{\mathbf{Q} \in \mathbf{R}^{3 \times 3}, \operatorname{tr}(\mathbf{Q})=0, \mathbf{Q}=\mathbf{Q}^{T}\right\}
$$

and consider the general Landau-De Gennes free energy functional

$$
\mathbf{E}(\mathbf{Q})=\int_{\Omega}\left(\frac{K}{2}|\nabla \mathbf{Q}|^{2}+F_{B}(\mathbf{Q})\right) d \mathbf{x}
$$

where the first term in the integral is the elastic energy, $K$ a material-dependent elastic constant, $F_{B}(\mathbf{Q})$ the bulk free energy density,

$$
F_{B}(\mathbf{Q}):=\frac{\alpha}{2} \operatorname{tr}\left(\mathbf{Q}^{2}\right)+\frac{\beta}{3} \operatorname{tr}\left(\mathbf{Q}^{3}\right)+\frac{\gamma}{4} \operatorname{tr}^{2}\left(\mathbf{Q}^{2}\right)
$$

and $\alpha, \beta$ and $\gamma>0$ are material-dependent and temperature-dependent constants - cf. [7].

According to $[1,20,23]$, the non-dimensional governing equations of nematic liquid crystal flows with hydrodynamics have the form

$$
\begin{aligned}
& \mathbf{u}_{t}+\mathbf{u} \cdot \nabla \mathbf{u}=-\nabla p+\eta \nabla^{2} \mathbf{u}+\nabla \cdot \sigma(\mathbf{Q}, \mathbf{G})-\mathbf{G} \nabla \mathbf{Q}, \\
& \nabla \cdot \mathbf{u}=0 \\
& \mathbf{Q}_{t}+\mathbf{u} \cdot \nabla \mathbf{Q}-S(\nabla \mathbf{u}, \mathbf{Q})=M_{1} \mathbf{G}
\end{aligned}
$$

where

$$
\begin{aligned}
& S(\nabla \mathbf{u}, \mathbf{Q})=W \cdot \mathbf{Q}-\mathbf{Q} \cdot W+a(\mathbf{Q} \cdot D+D \cdot \mathbf{Q})+\frac{2 a}{3}\left(D-\frac{\nabla \cdot \mathbf{u} I}{3}\right)-2 a(D: \mathbf{Q})\left(\mathbf{Q}+\frac{I}{3}\right) \\
& \sigma(\mathbf{Q}, \mathbf{G})=(\mathbf{Q} \cdot \mathbf{G}-\mathbf{G} \cdot \mathbf{Q})-a(\mathbf{G} \cdot \mathbf{Q}+\mathbf{Q} \cdot \mathbf{G})-\frac{2 a}{3} \mathbf{G}+2 a(\mathbf{Q}: \mathbf{G})\left(\mathbf{Q}+\frac{I}{3}\right) \\
& \mathbf{G}=-\frac{\delta \mathbf{E}(\mathbf{Q})}{\delta \mathbf{Q}}=K \nabla^{2} \mathbf{Q}-\left[\alpha \mathbf{Q}+\beta\left(\mathbf{Q}^{2}-\frac{\operatorname{tr}\left(\mathbf{Q}^{2}\right)}{3} \mathbf{I}\right)+\gamma \operatorname{tr}\left(\mathbf{Q}^{2}\right) \mathbf{Q}\right]
\end{aligned}
$$

and $D=\left(\nabla \mathbf{u}+\nabla \mathbf{u}^{T}\right) / 2$ and $W=\nabla \mathbf{u}-\nabla \mathbf{u}^{T} / 2$ are, respectively, the rate of strain and vorticity tensors. Moreover, the first two terms in $\mathbf{S}(\nabla \mathbf{u}, \mathbf{Q})$ and the material derivative of $\mathbf{Q}$ define the Gordon-Schowalter derivative, $\mathbf{G}$ is the molecular field, $\sigma(\mathbf{Q}, \mathbf{G})$ the elastic stress tensor, and $a \in[-1,1]$ a geometric parameter of the nematic liquid crystal molecule — cf. [20]. We also impose the initial condition

$$
\mathbf{u}(\mathbf{x}, 0)=\mathbf{u}_{0}(\mathbf{x}), \quad \mathbf{Q}(\mathbf{x}, 0)=\mathbf{Q}_{0}(\mathbf{x}),
$$

and use one of the following boundary conditions:

1. $\mathbf{u}$ and $\mathbf{Q}$ are periodic on $\partial \Omega$.

2. $\left.\mathbf{u}\right|_{\partial \Omega}=0,\left.\mathbf{Q}\right|_{\partial \Omega}=\mathbf{Q}^{0}$ or $\left.\partial_{n} \mathbf{Q}\right|_{\partial \Omega}=0$. 
This model is widely used in liquid crystal flows $[2,7,12,17,20,22]$. The well-posedness of the $\mathbf{Q}$-tensor model has been studied in $[4,8,9,13]$ and numerical approaches are discussed in $[11,14]$. In the framework of the model $(1.2)$, a variety of numerical codes have been developed, yet there are no systematic efforts to analyse the variational and dissipative nature of the method. The governing system (1.2) obeys the energy dissipation law [13] and it is desirable to develop numerical schemes with the same property. Such numerical schemes are called the energy-stable schemes. The main challenge in this approach to the Q-tensor model is caused by the high nonlinearity of the bulk potential $F_{B}$ and the strong coupling between the velocity field $\mathbf{u}$ and $\mathbf{Q}$ in the nonlinear stress term $\sigma$. Zhao and Wang [23] employed a stabiliser in decoupled first-order and coupled second-order energystable schemes. Their main result is based on the assumption that the Hessian matrix of the bulk potential function $F_{B}$ is bounded from above. Additionally, in the decoupled firstorder scheme, there is an artificially added term of order $\delta t$. Zhao et al. [24] employed an "IEQ" strategy in a linear second-order coupled energy-stable scheme, but the proof of the main result has a gap. Here, a linear coupled unconditionally energy-stable scheme is constructed by following the "SAV" strategy from $[15,16]$.

The rest of this paper is organised as follows. In Section 2, we derive a semi-discrete unconditionally energy-stable scheme and prove that the solution of the corresponding system is unique. Section 3 presents numerical examples to verify analytical results from Section 2. Our conclusions are in Section 4.

\section{A Second-Order Linear Unconditionally Energy-Stable Scheme}

Since the bulk energy $F_{B}(\mathbf{Q})$ is bounded from below, there is a constant $C_{0}$ such that

$$
\mathscr{E}_{1}=\int_{\Omega} F_{B} d \mathbf{x}+C_{0}>0
$$

Set $r=\sqrt{\mathscr{E}_{1}(\mathbf{Q})}$ and write the Eq. (1.3) in the form

$$
\begin{aligned}
& \mathbf{G}=\nabla \cdot(K \nabla \mathbf{Q})-\frac{r}{\sqrt{\mathscr{E}_{1}(\mathbf{Q})}} f_{B}(\mathbf{Q}), \\
& r_{t}=\frac{f_{B}(\mathbf{Q})}{2 \sqrt{\mathscr{E}_{1}(\mathbf{Q})}}: \mathbf{Q}_{t}, \\
& f_{B}(\mathbf{Q})=\alpha \mathbf{Q}+\beta\left(\mathbf{Q}^{2}-\frac{1}{3} \operatorname{tr}\left(\mathbf{Q}^{2}\right) \mathbf{I}\right)+\gamma \operatorname{tr}\left(\mathbf{Q}^{2}\right) \mathbf{Q} .
\end{aligned}
$$

The total energy (1.1) can be rewritten as

$$
\mathscr{E}=\int_{\Omega}\left(\frac{\mathbf{u}^{2}}{2}+\frac{K}{2}|\nabla \mathbf{Q}|^{2}\right) d \mathbf{x}+r^{2}-C_{0} .
$$

For the model (1.2) with (2.1)-(2.3), we now consider a second-order scheme based on the backward differentiation formula with Adam-Bashforth explicit interpolation (BDF2) [21]. 
More exactly, we set $\bar{f}^{n+1}=2 f^{n}-f^{n-1}$ and proceed as follows.

Step 1. Determine $\left(\mathbf{Q}^{n+1}, r^{n+1}, \tilde{\mathbf{u}}^{n+1}\right)$ from the equations

$$
\begin{aligned}
& \frac{3 \mathbf{Q}^{n+1}-4 \mathbf{Q}^{n}+\mathbf{Q}^{n-1}}{2 \delta t}+\left(\tilde{\mathbf{u}}^{n+1} \cdot \nabla\right) \overline{\mathbf{Q}}^{n+1}-S\left(\nabla \tilde{\mathbf{u}}^{n+1}, \overline{\mathbf{Q}}^{n+1}\right)=M_{1} \mathbf{G}^{n+1}, \\
& \frac{3 \tilde{\mathbf{u}}^{n+1}-4 \mathbf{u}^{n}+\mathbf{u}^{n-1}}{2 \delta t}+\left(\overline{\mathbf{u}}^{n+1} \cdot \nabla\right) \tilde{\mathbf{u}}^{n+1}=\eta \triangle \tilde{\mathbf{u}}^{n+1}-\nabla p^{n} \\
& \quad+\nabla \cdot \sigma\left(\overline{\mathbf{Q}}^{n+1}, \mathbf{G}^{n+1}\right)-\nabla \overline{\mathbf{Q}}^{n+1}: \mathbf{G}^{n+1},
\end{aligned}
$$

where

$$
\begin{aligned}
& \mathbf{G}^{n+1}=\nabla \cdot\left(K \nabla \mathbf{Q}^{n+1}\right)-\frac{f_{B}\left(\overline{\mathbf{Q}}^{n+1}\right)}{\sqrt{\mathscr{E}_{1}\left(\overline{\mathbf{Q}}^{n+1}\right)}} r^{n+1}, \\
& 3 r^{n+1}-4 r^{n}+r^{n-1}=\int_{\Omega} \frac{f_{B}\left(\overline{\mathbf{Q}}^{n+1}\right)}{2 \sqrt{\mathscr{E}_{1}\left(\overline{\mathbf{Q}}^{n+1}\right)}}:\left(3 \mathbf{Q}^{n+1}-4 \mathbf{Q}^{n}+\mathbf{Q}^{n-1}\right) d \mathbf{x} .
\end{aligned}
$$

Step 2. Find $\left(\mathbf{u}^{n+1}, p^{n+1}\right)$ from the equations

$$
\begin{aligned}
& 3 \frac{\mathbf{u}^{n+1}-\tilde{\mathbf{u}}^{n+1}}{2 \delta t}+\nabla\left(p^{n+1}-p^{n}\right)=0, \\
& \nabla \cdot \mathbf{u}^{n+1}=0
\end{aligned}
$$

with boundary condition $\left.\mathbf{u}^{n+1} \cdot \mathbf{n}\right|_{\partial \Omega}=0$.

Let us note the representation

$$
\begin{aligned}
2\left(a^{n+1}, 3 a^{n+1}-4 a^{n}+a^{n-1}\right)= & \left|a^{n+1}\right|^{2}+\left|2 a^{n+1}-a^{n}\right|^{2}+\left|a^{n+1}-2 a^{n}+a^{n-1}\right|^{2} \\
& -\left|a^{n}\right|^{2}-\left|2 a^{n}-a^{n-1}\right|^{2}
\end{aligned}
$$

used in the proof of the following theorem.

Theorem 2.1. The scheme (2.4)-(2.9) is unconditionally energy stable and satisfies the semidiscrete energy dissipation law

$$
\mathscr{E}_{D B F 2}^{n+1}-\mathscr{E}_{D B F 2}^{n} \leq-\delta t\left(M_{1}\left\|\mathbf{G}^{n+1}\right\|^{2}+\eta\left\|\nabla \tilde{\mathbf{u}}^{n+1}\right\|^{2}\right),
$$

where

$$
\begin{aligned}
\mathscr{E}_{B D F 2}^{n+1}= & \frac{1}{2}\left(\frac{\left\|\mathbf{u}^{n+1}\right\|^{2}}{2}+\frac{\left\|2 \mathbf{u}^{n+1}-\mathbf{u}^{n}\right\|^{2}}{2}\right)+\frac{K}{2}\left(\frac{\left\|\nabla \mathbf{Q}^{n+1}\right\|^{2}}{2}+\frac{\left\|2 \nabla \mathbf{Q}^{n+1}-\nabla \mathbf{Q}^{n}\right\|^{2}}{2}\right) \\
& +\left(\frac{\left|r^{n+1}\right|^{2}}{2}+\frac{\left|2 r^{n+1}-r^{n}\right|^{2}}{2}\right)+\frac{\delta t^{2}}{3}\left\|\nabla p^{n}\right\|^{2} .
\end{aligned}
$$

Proof. We consider the $L^{2}$-inner products of the Eq. (2.4) with $2 \delta t \mathbf{G}^{n+1}$ and the (2.6) with $3 \mathbf{Q}^{n+1}-4 \mathbf{Q}^{n}+\mathbf{Q}^{n-1}$. Multiplying (2.7) and $2 r^{n+1}$, and using the identity (2.10), we obtain

$$
\left(3 \mathbf{Q}^{n+1}-4 \mathbf{Q}^{n}+\mathbf{Q}^{n-1}, \mathbf{G}^{n+1}\right)+2 \delta t\left(\left(\tilde{\mathbf{u}}^{n+1} \cdot \nabla\right) \overline{\mathbf{Q}}^{n}, \mathbf{G}^{n+1}\right)
$$


A BDF2 Energy-Stable Scheme

$$
\begin{aligned}
& -2 \delta t\left(S\left(\nabla \tilde{\mathbf{u}}^{n+1}, \overline{\mathbf{Q}}^{n}\right), \mathbf{G}^{n+1}\right)=2 \delta t M_{1}\left\|\mathbf{G}^{n+1}\right\|^{2}, \\
& \left(\mathbf{G}^{n+1}, 3 \mathbf{Q}^{n+1}-4 \mathbf{Q}^{n}+\mathbf{Q}^{n-1}\right) \\
= & -r^{n+1} \int_{\Omega} \frac{f_{B}\left(\overline{\mathbf{Q}}^{n+1}\right)}{\sqrt{\mathscr{E}_{1}\left(\overline{\mathbf{Q}}^{n+1}\right)}:\left(3 \mathbf{Q}^{n+1}-4 \mathbf{Q}^{n}+\mathbf{Q}^{n-1}\right) d \mathbf{x}} \\
& -K\left(\frac{\left\|\nabla \mathbf{Q}^{n+1}\right\|^{2}+\left\|2 \nabla \mathbf{Q}^{n+1}-\nabla \mathbf{Q}^{n}\right\|^{2}+\left\|\nabla \mathbf{Q}^{n+1}-2 \nabla \mathbf{Q}^{n}+\nabla \mathbf{Q}^{n-1}\right\|^{2}}{2}\right. \\
& \left.-\frac{\left\|\nabla \mathbf{Q}^{n}\right\|^{2}+\left\|2 \nabla \mathbf{Q}^{n}-\nabla \mathbf{Q}^{n-1}\right\|^{2}}{2}\right) \\
& \times 2\left(\frac{\left|r^{n+1}\right|^{2}+\left|2 r^{n+1}-r^{n}\right|^{2}+\left|r^{n+1}-2 r^{n}+r^{n-1}\right|^{2}}{2}-\frac{\left|r^{n}\right|^{2}+\left|2 r^{n}-r^{n-1}\right|^{2}}{2}\right) \\
= & r^{n+1} \int_{\Omega} \frac{f_{B}\left(\overline{\mathbf{Q}}^{n+1}\right)}{\sqrt{\mathscr{E}_{1}\left(\overline{\mathbf{Q}}^{n+1}\right)}:\left(3 \mathbf{Q}^{n+1}-4 \mathbf{Q}^{n}+\mathbf{Q}^{n-1}\right) d \mathbf{x} .}
\end{aligned}
$$

In follows from the three equations above that

$$
\begin{aligned}
& K\left(\frac{\left\|\nabla \mathbf{Q}^{n+1}\right\|^{2}+\left\|2 \nabla \mathbf{Q}^{n+1}-\nabla \mathbf{Q}^{n}\right\|^{2}+\left\|\nabla \mathbf{Q}^{n+1}-2 \nabla \mathbf{Q}^{n}+\nabla \mathbf{Q}^{n-1}\right\|^{2}}{2}\right. \\
& \left.-\frac{\left\|\nabla \mathbf{Q}^{n}\right\|^{2}+\left\|2 \nabla \mathbf{Q}^{n}-\nabla \mathbf{Q}^{n-1}\right\|^{2}}{2}\right) \\
& +2\left(\frac{\left|r^{n+1}\right|^{2}+\left|2 r^{n+1}-r^{n}\right|^{2}+\left|r^{n+1}-2 r^{n}+r^{n-1}\right|^{2}}{2}-\frac{\left|r^{n}\right|^{2}+\left|2 r^{n}-r^{n-1}\right|^{2}}{2}\right) \\
& -2 \delta t\left(\left(\tilde{\mathbf{u}}^{n+1} \cdot \nabla\right) \overline{\mathbf{Q}}^{n+1}, \mathbf{G}^{n+1}\right)+2 \delta t\left(S\left(\nabla \tilde{\mathbf{u}}^{n+1}, \overline{\mathbf{Q}}^{n+1}\right), \mathbf{G}^{n+1}\right) \\
& =-2 \delta t M_{1}\left\|\mathbf{G}^{n+1}\right\|^{2} .
\end{aligned}
$$

Consider now the $L^{2}$-inner products of (2.5) with $2 \delta t \tilde{\mathbf{u}}^{n+1}$ and (2.8) with $\mathbf{u}^{n+1}$, i.e.

$$
\begin{aligned}
& \left(3 \tilde{\mathbf{u}}^{n+1}-4 \mathbf{u}^{n}+\mathbf{u}^{n-1}, \tilde{\mathbf{u}}^{n+1}\right)=-2 \delta t \eta\left\|\nabla \tilde{\mathbf{u}}^{n+1}\right\|^{2}-2 \delta t\left(\nabla p^{n}, \tilde{\mathbf{u}}^{n+1}\right) \\
& \quad+2 \delta t\left(\nabla \cdot \sigma\left(\overline{\mathbf{Q}}^{n}, \mathbf{G}^{n+1}\right), \tilde{\mathbf{u}}^{n+1}\right)-2 \delta t\left(\nabla \overline{\mathbf{Q}}^{n}: \mathbf{G}^{n+1}, \tilde{\mathbf{u}}^{n+1}\right), \\
& \frac{3}{4 \delta t}\left(\left\|\mathbf{u}^{n+1}\right\|^{2}+\left\|\mathbf{u}^{n+1}-\tilde{\mathbf{u}}^{n+1}\right\|^{2}-\left\|\tilde{\mathbf{u}}^{n+1}\right\|^{2}\right)=0 .
\end{aligned}
$$

The Eq. (2.8) shows that for any divergence-free function $\mathbf{v}$ we have

$$
\left(\mathbf{u}^{n+1}, \mathbf{v}\right)=\left(\tilde{\mathbf{u}}^{n+1}, \mathbf{v}\right) \text {. }
$$

The left-hand side of (2.14) can be written as

$$
\begin{aligned}
& \left(3 \tilde{\mathbf{u}}^{n+1}-4 \mathbf{u}^{n}+\mathbf{u}^{n-1}, \tilde{\mathbf{u}}^{n+1}\right) \\
= & \left(3 \tilde{\mathbf{u}}^{n+1}-3 \mathbf{u}^{n+1}, \tilde{\mathbf{u}}^{n+1}\right)+\left(3 \mathbf{u}^{n+1}-4 \mathbf{u}^{n}+\mathbf{u}^{n-1}, \tilde{\mathbf{u}}^{n+1}\right) \\
= & \left(3 \tilde{\mathbf{u}}^{n+1}-3 \mathbf{u}^{n+1}, \tilde{\mathbf{u}}^{n+1}\right)+\left(3 \mathbf{u}^{n+1}-4 \mathbf{u}^{n}+\mathbf{u}^{n-1}, \mathbf{u}^{n+1}\right)
\end{aligned}
$$




$$
\begin{aligned}
= & \left(3 \tilde{\mathbf{u}}^{n+1}-3 \mathbf{u}^{n+1}, \tilde{\mathbf{u}}^{n+1}+\mathbf{u}^{n+1}\right)+\left(3 \tilde{\mathbf{u}}^{n+1}-3 \mathbf{u}^{n+1}, \mathbf{u}^{n+1}\right) \\
& +\left(3 \mathbf{u}^{n+1}-4 \mathbf{u}^{n}+\mathbf{u}^{n-1}, \mathbf{u}^{n+1}\right) \\
= & 3\left(\left\|\tilde{\mathbf{u}}^{n+1}\right\|^{2}-\left\|\mathbf{u}^{n+1}\right\|^{2}\right) \\
& +\frac{\left\|\mathbf{u}^{n+1}\right\|^{2}+\left\|2 \mathbf{u}^{n+1}-\mathbf{u}^{n}\right\|^{2}+\left\|\mathbf{u}^{n+1}-2 \mathbf{u}^{n}+\mathbf{u}^{n-1}\right\|^{2}}{2} \\
& -\frac{\left\|\mathbf{u}^{n}\right\|^{2}+\left\|2 \mathbf{u}^{n}-\mathbf{u}^{n-1}\right\|^{2}}{2} .
\end{aligned}
$$

Besides, we represent the Eq. (2.8) in the form

$$
\frac{3}{2 \delta t} \mathbf{u}^{n+1}+\nabla p^{n+1}=\frac{3}{2 \delta t} \tilde{\mathbf{u}}^{n+1}+\nabla p^{n} .
$$

Squaring this equation and multiplying the result by $\delta t / 3$ yields

$$
\frac{3}{4 \delta t}\left(\left\|\mathbf{u}^{n+1}\right\|^{2}-\left\|\tilde{\mathbf{u}}^{n+1}\right\|^{2}\right)+\frac{\delta t}{3}\left(\left\|\nabla p^{n+1}\right\|^{2}-\left\|\nabla p^{n}\right\|^{2}\right)=\left(\tilde{\mathbf{u}}^{n+1}, \nabla p^{n}\right) .
$$

Combining the Eqs. (2.14)-(2.18), we obtain

$$
\begin{aligned}
& \frac{\left\|\mathbf{u}^{n+1}\right\|^{2}+\left\|2 \mathbf{u}^{n+1}-\mathbf{u}^{n}\right\|^{2}}{2}-\frac{\left\|\mathbf{u}^{n}\right\|^{2}+\left\|2 \mathbf{u}^{n}-\mathbf{u}^{n-1}\right\|^{2}}{2} \\
& +\frac{2 \delta t^{2}}{3}\left(\left\|\nabla p^{n+1}\right\|^{2}-\left\|\nabla p^{n}\right\|^{2}\right)+\frac{3}{2}\left\|\mathbf{u}^{n+1}-\tilde{\mathbf{u}}^{n+1}\right\|^{2} \\
& +\frac{\left\|\mathbf{u}^{n+1}-2 \mathbf{u}^{n}+\mathbf{u}^{n-1}\right\|^{2}}{2} \\
= & -2 \delta t \eta\left\|\nabla \tilde{\mathbf{u}}^{n+1}\right\|^{2}+2 \delta t\left(\nabla \cdot \sigma\left(\overline{\mathbf{Q}}^{n+1}, \mathbf{G}^{n+1}\right), \tilde{\mathbf{u}}^{n+1}\right) \\
& -2 \delta t\left(\nabla \overline{\mathbf{Q}}^{n+1}: \mathbf{G}^{n+1}, \tilde{\mathbf{u}}^{n+1}\right) .
\end{aligned}
$$

The inequality (2.11) now follows from (2.12), (2.13) and (2.19).

The Eqs. (2.4)-(2.7) show that the system in Step 1 is linear with respect to unknowns $\mathbf{Q}^{n+1}$ and $\tilde{\mathbf{u}}^{n+1}$. We prove that the solution of this system is unique. Indeed, let us assume that for given $\mathbf{Q}^{n}, \mathbf{Q}^{n-1}, \mathbf{u}^{n}$ and $\mathbf{u}^{n-1}$ the system has two solutions $\left(\mathbf{Q}_{1}, \tilde{\mathbf{u}}_{1}\right)$ and $\left(\mathbf{Q}_{2}, \tilde{\mathbf{u}}_{2}\right)$. Considering the terms $\mathbf{Q}_{0}=\mathbf{Q}_{1}-\mathbf{Q}_{2}$ and $\tilde{\mathbf{u}}_{0}=\tilde{\mathbf{u}}_{1}-\tilde{\mathbf{u}}_{2}$, we have

$$
\begin{aligned}
& \frac{3 \mathbf{Q}_{0}}{2 \delta t}+\left(\tilde{\mathbf{u}}_{0} \cdot \nabla\right) \overline{\mathbf{Q}}^{n+1}-S\left(\nabla \tilde{\mathbf{u}}_{0}, \overline{\mathbf{Q}}^{n+1}\right)=M_{1} \mathbf{G}_{0}, \\
& \frac{3 \tilde{\mathbf{u}}_{0}}{2 \delta t}+\left(\overline{\mathbf{u}}^{n+1} \cdot \nabla\right) \tilde{\mathbf{u}}_{0}=\eta \triangle \tilde{\mathbf{u}}_{0}+\nabla \cdot \sigma\left(\overline{\mathbf{Q}}^{n+1}, \mathbf{G}_{0}\right)-\nabla \overline{\mathbf{Q}}^{n+1}: \mathbf{G}_{0},
\end{aligned}
$$

where

$$
\begin{aligned}
\mathbf{G}_{0} & =K \triangle \mathbf{Q}_{0}-\frac{f_{B}\left(\overline{\mathbf{Q}}^{n+1}\right)}{\sqrt{\mathscr{E}_{1}\left(\overline{\mathbf{Q}}^{n+1}\right)}} r_{0}, \\
r_{0} & =\int_{\Omega} \frac{f_{B}\left(\overline{\mathbf{Q}}^{n+1}\right)}{2 \sqrt{\mathscr{E}_{1}\left(\overline{\mathbf{Q}}^{n+1}\right)}}: \mathbf{Q}_{0} d \mathbf{x} .
\end{aligned}
$$


Computing the $L^{2}$-inner products of (2.20) with $\mathbf{G}_{0}$, (2.22) with $\mathbf{Q}_{0}$ and (2.21) with $\tilde{\mathbf{u}}_{0}$, and using the relationship (2.23), we obtain

$$
\begin{aligned}
& \frac{3}{2 \delta t}\left(\mathbf{Q}_{0}, \mathbf{G}_{0}\right)+\left(\tilde{\mathbf{u}}_{0} \cdot \nabla \overline{\mathbf{Q}}^{n+1}, \mathbf{G}_{0}\right)-\left(S\left(\nabla \tilde{\mathbf{u}}_{0}, \overline{\mathbf{Q}}^{n+1}\right), \mathbf{G}_{0}\right)=M_{1}\left\|\mathbf{G}_{0}\right\|^{2}, \\
& \left(\mathbf{G}_{0}, \mathbf{Q}_{0}\right)=-K\left\|\nabla \mathbf{Q}_{0}\right\|^{2}-2\left|r_{0}\right|^{2}, \\
& \frac{3}{2 \delta t}\left\|\tilde{\mathbf{u}}_{0}\right\|^{2}=-\eta\left\|\nabla \tilde{\mathbf{u}}_{0}\right\|^{2}+\left(\nabla \cdot \sigma\left(\overline{\mathbf{Q}}^{n+1}, \mathbf{G}_{0}\right), \tilde{\mathbf{u}}_{0}\right)-\left(\nabla \overline{\mathbf{Q}}^{n+1}: \mathbf{G}_{0}, \tilde{\mathbf{u}}_{0}\right) .
\end{aligned}
$$

The three equations above yield

$$
\frac{3\left\|\tilde{\mathbf{u}}_{0}\right\|^{2}}{2 \delta t}+2\left|r_{0}\right|^{2}+M_{1}\left\|\mathbf{G}_{0}\right\|^{2}+K\left\|\nabla \mathbf{Q}_{0}\right\|^{2}+\left\|\tilde{\mathbf{u}}_{0}\right\|^{2}=0,
$$

so that $\mathbf{Q}_{0}=0, \tilde{\mathbf{u}}_{0}=0$.

Applying the Eq. (2.7), we eliminate the term $r^{n+1}$ in (2.13), thus obtaining

$$
\left(\frac{3}{2 \delta t}-M_{1} K \triangle\right) \mathbf{Q}^{n+1}+\frac{M_{1}}{2} \overline{\mathbf{H}}^{n+1} \int_{\Omega} \overline{\mathbf{H}}^{n+1}: \mathbf{Q}^{n+1} d \mathbf{x}+\mathscr{L}_{1}\left(\tilde{\mathbf{u}}^{n+1}\right)=\mathbf{g}_{1}^{n},
$$

where

$$
\begin{aligned}
& \mathscr{L}_{1}\left(\tilde{\mathbf{u}}^{n+1}\right)=\left(\tilde{\mathbf{u}}^{n+1} \cdot \nabla\right) \overline{\mathbf{Q}}^{n+1}-S\left(\nabla \tilde{\mathbf{u}}^{n+1}, \overline{\mathbf{Q}}^{n+1}\right) \\
& \overline{\mathbf{H}}^{n+1}=\frac{f_{B}\left(\overline{\mathbf{Q}}^{n+1}\right)}{\sqrt{\mathscr{E}_{1}\left(\overline{\mathbf{Q}}^{n+1}\right)}}, \\
& \mathbf{g}_{1}^{n}=\frac{4 \mathbf{Q}^{n}-\mathbf{Q}^{n-1}}{2 \delta t}-\frac{M_{1}\left(4 r^{n}-r^{n-1}\right)}{3} \overline{\mathbf{H}}^{n+1}+\frac{M_{1}}{6} \overline{\mathbf{H}}^{n+1} \int_{\Omega} \overline{\mathbf{H}}^{n+1}:\left(4 \mathbf{Q}^{n}-\mathbf{Q}^{n-1}\right) d \mathbf{x} .
\end{aligned}
$$

Introducing the notation

$$
b^{n+1}=\int_{\Omega} \overline{\mathbf{H}}^{n+1}: \mathbf{Q}^{n+1} d \mathbf{x}, \mathscr{L}=\left(\frac{3}{2 \delta t}-M_{1} K \triangle\right),
$$

we write the Eq. (2.24) as

$$
\mathbf{Q}^{n+1}+\frac{M_{1} b^{n+1}}{2} \mathscr{L}^{-1} \overline{\mathbf{H}}^{n+1}=\mathscr{L}^{-1}\left(\mathbf{g}_{1}^{n}-\mathscr{L}_{1}\left(\tilde{\mathbf{u}}^{n+1}\right)\right) .
$$

In order to determine the term $b^{n+1}$, we compute the inner product of (2.25) and $\overline{\mathbf{H}}^{n+1}$. This leads to the representation

$$
b^{n+1}=\left(\int_{\Omega} \overline{\mathbf{H}}^{n+1}: \mathscr{L}^{-1}\left(\mathbf{g}_{1}^{n}-\mathscr{L}_{1}\left(\tilde{\mathbf{u}}^{n+1}\right)\right) d \mathbf{x}\right) /\left(\left(1+M_{1} / 2\right) \int_{\Omega} \overline{\mathbf{H}}^{n+1}: \mathscr{L}^{-1} \overline{\mathbf{H}}^{n+1} d \mathbf{x}\right) .
$$

Now we set

$$
\mathscr{L}_{2}\left(\mathbf{G}^{n+1}\right):=\nabla \overline{\mathbf{Q}}^{n+1}: \mathbf{G}^{n+1}-\nabla \cdot \sigma\left(\overline{\mathbf{Q}}^{n+1}, \mathbf{G}^{n+1}\right)
$$




$$
\mathbf{g}_{2}^{n}:=\frac{4 \mathbf{u}^{n}-\mathbf{u}^{n-1}}{2 \delta t}-\nabla p^{n}
$$

and write the Eq. (2.5) as

$$
\frac{3 \tilde{\mathbf{u}}^{n+1}}{2 \delta t}+\left(\overline{\mathbf{u}}^{n+1} \cdot \nabla\right) \tilde{\mathbf{u}}^{n+1}-\eta \triangle \tilde{\mathbf{u}}^{n+1}=\mathbf{g}_{2}^{n}-\mathscr{L}_{2}\left(\mathbf{G}^{n+1}\right) .
$$

Step 1 in the above numerical scheme is equivalent to the Eqs. (2.25)-(2.27). In order to decouple $\mathbf{Q}^{n+1}$ and $\tilde{\mathbf{u}}^{n+1}$, we now use the Gauss-Seidel type iterations, so that

$$
\begin{aligned}
& \mathbf{Q}^{n+1, k+1}=\mathscr{L}^{-1}\left(\mathbf{g}_{1}-\mathscr{L}_{1}\left(\tilde{\mathbf{u}}^{n+1, k}\right)\right)-\frac{M_{1} b^{n+1, k}}{2} \mathscr{L}^{-1} \overline{\mathbf{H}}^{n+1}, \\
& b^{n+1, k}=\frac{\int_{\Omega} \overline{\mathbf{H}}^{n+1}: \mathscr{L}^{-1}\left(\mathbf{g}_{1}^{n}-\mathscr{L}_{1}\left(\tilde{\mathbf{u}}^{n+1, k}\right)\right) d \mathbf{x}}{\left(1+M_{1} / 2\right) \int_{\Omega} \overline{\mathbf{H}}^{n+1}: \mathscr{L}^{-1} \overline{\mathbf{H}}^{n+1} d \mathbf{x}}, \\
& \frac{3 \tilde{\mathbf{u}}^{n+1, k+1}}{2 \delta t}+B\left(\overline{\mathbf{u}}^{n+1}, \tilde{\mathbf{u}}^{n+1, k+1}\right)-\eta \triangle \tilde{\mathbf{u}}^{n+1, k+1}=\mathbf{g}_{2}^{n}-\mathscr{L}_{2}\left(\mathbf{G}^{n+1, k+1}\right) .
\end{aligned}
$$

\section{Numerical Results}

In numerical simulations below, we assume that the directors of the LCPs are imposed in the $x-y$ plane, so that the tensor $Q$ is a $2 \times 2$ matrix. The unknown functions depend on variables $x$ and $y$ only and the model parameters are

$$
a=1.0, \quad \eta=1, \quad \alpha=-0.2, \quad \gamma=1, \quad K=0.001, \quad M_{1}=1, \quad C_{0}=10 .
$$

\subsection{Accuracy test}

In the first example, we choose the initial and boundary conditions

$$
\begin{aligned}
& \mathbf{u}_{0}=0, \quad \mathbf{Q}_{0}=\mathbf{n}_{\mathbf{0}} \mathbf{n}_{\mathbf{0}}^{T}-\frac{\left\|\mathbf{n}_{\mathbf{0}}\right\|^{\mathbf{2}}}{2} \\
& \left.\mathbf{u}\right|_{\partial \Omega}=0,\left.\quad \mathbf{Q}\right|_{\partial \Omega}=\left.\mathbf{Q}_{0}\right|_{\partial \Omega},
\end{aligned}
$$

where $\mathbf{n}_{\mathbf{0}}=(\sin (2 \pi x) \sin (2 \pi y), 0)^{T}$. Additional data $\mathbf{u}_{1}, \mathbf{Q}_{1}$ can be obtained by the firstorder scheme with a small time step. Avoiding the spatial discrete error, we employ $512 \times$ 512 mesh grid on $[0,1]^{2}$. The convergence order shown in Tables 1 and 2, approaches 2 if time step $\delta t$ tends to 0 , consistent with theoretical analysis.

In the second example, we choose the initial and boundary conditions

$$
\begin{aligned}
& \mathbf{u}_{0}=0, \quad \mathbf{Q}_{0}=\mathbf{n}_{0} \mathbf{n}_{0}^{T}-\frac{\mathbf{I}}{2}, \\
& \left.\mathbf{u}\right|_{\partial \Omega}=0,\left.\quad \mathbf{Q}\right|_{\partial \Omega}=\left.\mathbf{Q}_{0}\right|_{\partial \Omega},
\end{aligned}
$$

where $\mathbf{n}_{0}=(\cos 2 \pi x y, \sin 2 \pi x y)^{T}$ and consider the same computational domain and mesh size. 
Table 1: Convergence of the schemes, $t=1.0$.

\begin{tabular}{|c|c|c|c|c|c|c|c|c|}
\hline Time step & $L-2$ error & \multirow{2}{*}{ Order } & $L-2$ error & \multirow{2}{*}{ Order } & $L-2$ error & \multirow{2}{*}{ Order } & $L-2$ error & \multirow{2}{*}{ Order } \\
\hline$\delta t$ & $Q_{11}$ & & $Q_{12}$ & & $\mathrm{u}$ & & $\mathrm{V}$ & \\
\hline 0.02 & $2.87 e-4$ & & $1.20 \mathrm{e}-4$ & & $1.94 \mathrm{e}-6$ & & $1.96 \mathrm{e}-6$ & \\
\hline 0.01 & $8.13 e-5$ & 1.82 & $3.24 \mathrm{e}-5$ & 1.89 & $5.71 \mathrm{e}-7$ & 1.76 & $5.82 \mathrm{e}-7$ & 1.75 \\
\hline $5.0 \mathrm{e}-3$ & $1.96 e-5$ & 2.05 & $7.74 \mathrm{e}-6$ & 2.07 & $1.66 \mathrm{e}-7$ & 1.78 & $1.68 \mathrm{e}-7$ & 1.79 \\
\hline $2.5 e-3$ & $4.88 \mathrm{e}-6$ & 2.01 & $1.93 e-6$ & 2.00 & $4.17 \mathrm{e}-8$ & 1.99 & $4.19 \mathrm{e}-8$ & 2.00 \\
\hline
\end{tabular}

Table 2: Convergence of the schemes, $t=1.0$.

\begin{tabular}{||c|c|c|c|c|c|c|c|c||}
\hline Time step & $L-2$ error & \multirow{2}{*}{ Order } & $L-2$ error & \multirow{2}{*}{ Order } & $L-2$ error & \multirow{2}{*}{ Order } & $L-2$ error & \multirow{2}{*}{ Order } \\
& & $Q_{12}$ & & $\mathrm{u}$ & & $\mathrm{v}$ & \\
\hline 0.02 & $4.31 \mathrm{e}-4$ & & $6.36 \mathrm{e}-4$ & & $2.98 \mathrm{e}-5$ & & $2.79 \mathrm{e}-5$ & \\
\hline 0.01 & $1.32 \mathrm{e}-4$ & 1.71 & $1.67 \mathrm{e}-4$ & 1.93 & $5.65 \mathrm{e}-6$ & 2.40 & $5.59 \mathrm{e}-6$ & 2.32 \\
\hline $5.0 \mathrm{e}-3$ & $4.30 \mathrm{e}-5$ & 1.62 & $4.34 \mathrm{e}-5$ & 1.94 & $1.35 \mathrm{e}-6$ & 2.07 & $1.38 \mathrm{e}-6$ & 2.02 \\
\hline $2.5 \mathrm{e}-3$ & $1.24 \mathrm{e}-5$ & 1.80 & $1.13 \mathrm{e}-5$ & 1.94 & $3.36 \mathrm{e}-7$ & 2.01 & $3.42 \mathrm{e}-7$ & 2.01 \\
\hline $1.25 \mathrm{e}-3$ & $2.89 \mathrm{e}-6$ & 2.10 & $2.85 \mathrm{e}-6$ & 1.99 & $8.40 \mathrm{e}-8$ & 2.00 & $8.55 \mathrm{e}-8$ & 2.00 \\
\hline
\end{tabular}

\subsection{Development of the defects}

The next examples deal with the defect development. We start with the defect at the point +1 subject to the Dirichlet boundary condition on $\mathbf{Q}$. For the domain $\left[0, L_{x}\right] \times\left[0, L_{y}\right]$, $L_{x}=L_{y}=2$, we consider a no-slip boundary condition for $\mathbf{u},\left.\mathbf{u}\right|_{\partial \Omega}=0$ and a Dirichlet boundary condition for $\mathbf{Q}$, viz.

$$
\left.\mathbf{Q}\right|_{\partial \Omega}=\frac{\tilde{\mathbf{n}}_{0} \tilde{\mathbf{n}}_{0}^{T}}{\left\|\tilde{\mathbf{n}}_{0}\right\|^{2}}-\frac{\mathbf{I}}{2}, \quad \tilde{\mathbf{n}}_{0}=\left(x-0.5 L_{x}, y-0.5 L_{y}\right)^{T}
$$

and the initial value

$$
\mathbf{Q}_{0}=\frac{\mathbf{n}_{0} \mathbf{n}_{0}^{T}}{\left\|\mathbf{n}_{0}\right\|^{2}}-\frac{\mathbf{I}}{2}, \quad \mathbf{n}_{0}=\left(x-0.25 L_{x}, y-0.25 L_{y}\right)^{T} .
$$

Fig. 2 (a) shows an initial +1 type defect at the point $(0.25,0.25)$. The boundary condition breaks the initial defect into two $+1 / 2$ type defects moving away from each other and reaching a stable state. In Figs. 2(b)-2(e), the line segments indicate the director of the LC and the colors show the parameter orders. The order parameters at the defects are almost zero - cf. blue points in Figs. 2(b)-2(e). Fig. 1 shows that the system energy decreases with time, consistent with our analysis. The velocity at $t=50,100,200$ is shown in Figs. 2(f)2(h).

In this example, we use the initial data

$$
\mathbf{Q}_{0}=\frac{\mathbf{n}_{0} \mathbf{n}_{0}^{T}}{\left\|\mathbf{n}_{0}\right\|^{2}}-\frac{\mathbf{I}}{2}, \quad \mathbf{n}_{0}= \begin{cases}(1,0)^{T}, & \sqrt{(x-1)^{2}+(y-1)^{2}}<0.4, \\ (0,1)^{T}, & \text { otherwise }\end{cases}
$$




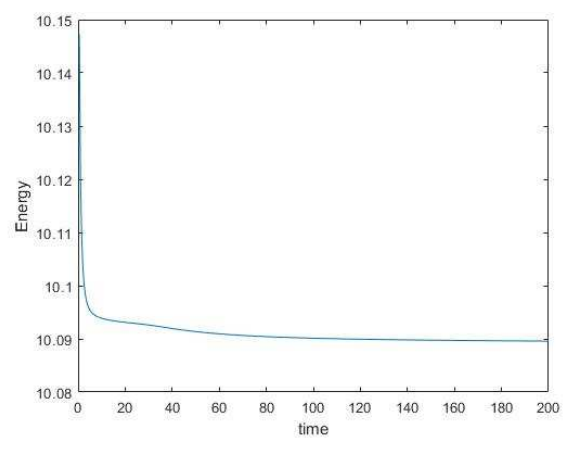

Energy

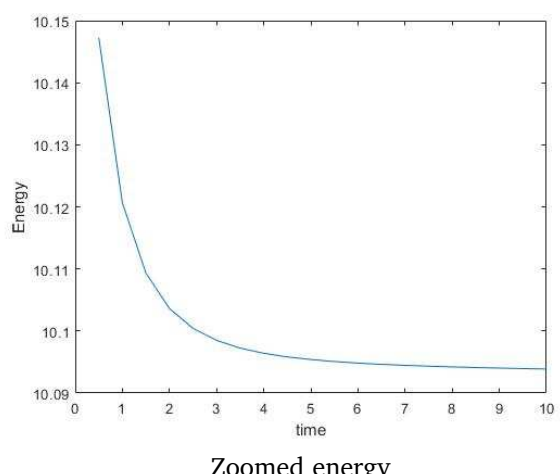

Figure 1: Energy versus time.

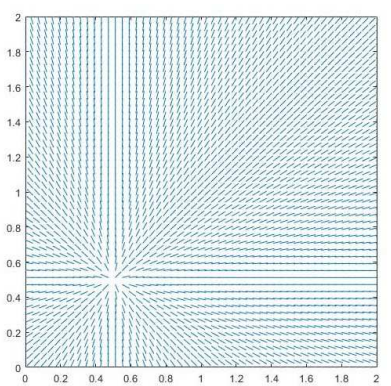

a)

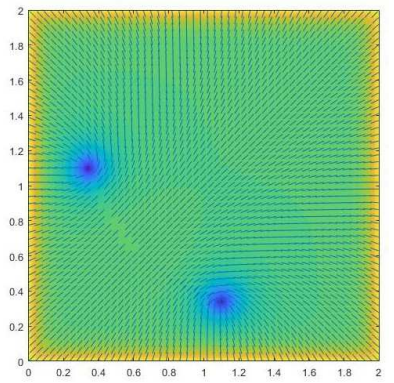

d)

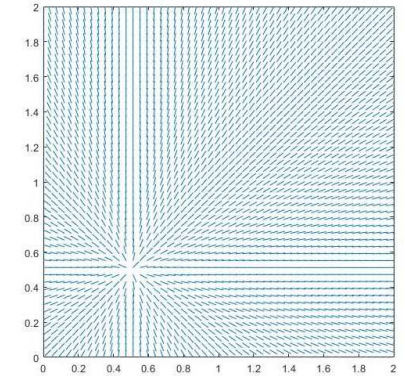

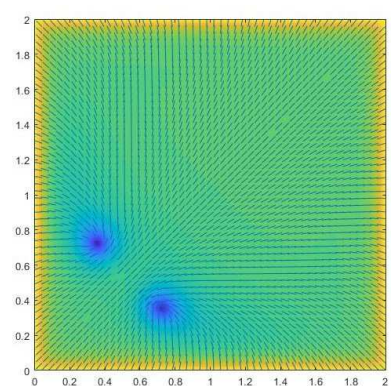

b)

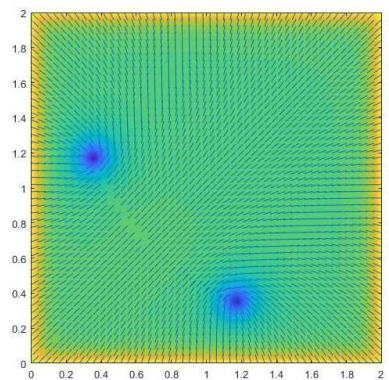

e)

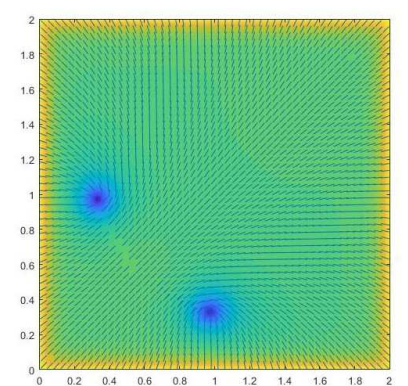

c)

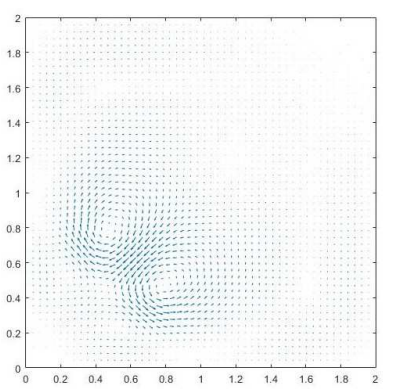

f)

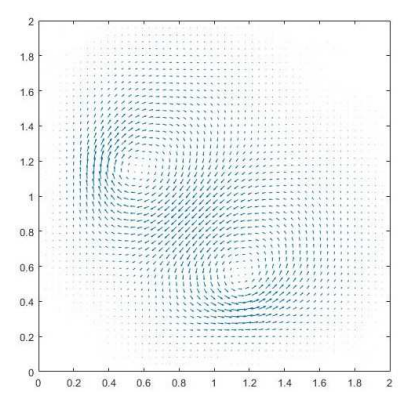

h)

Figure 2: (a) shows the director of initial data $\mathbf{Q}_{0}$; (b)-(e) show the director and order parameters (in color) for $t=50,100,150,200$, respectively; (f)-(h) show the velocity at $t=50,100,200$, respectively. 
and the boundary conditions

$$
\left.\mathbf{Q}\right|_{\partial \Omega}=\frac{\tilde{\mathbf{n}}_{0} \tilde{\mathbf{n}}_{0}^{T}}{\left\|\tilde{\mathbf{n}}_{0}\right\|^{2}}-\frac{\mathbf{I}}{2}, \quad \tilde{\mathbf{n}}_{0}=(0,1)^{T},\left.\mathbf{u}\right|_{\partial \Omega}=0
$$

We also consider the liquid crystal dynamics in situations with and without hydrodynamic effects for the same set of parameters and present the corresponding results in Figs. 3 and 4 , respectively.

There is a mismatch in orientation in the circular region initially. In Fig. 3, we use the line segment to indicate the directors and the colors to indicate the order parameters. The graphs show that the order parameters are close to 0 near the defect points (the blue points). The mismatch produces two $+1 / 2$ type defects (left and right) and two $-1 / 2$ type defects (top and bottom). Defects approach each other and disappear. The directors become homogeneous perpendicular to the $y$-boundary.

Fig. 4 shows that the radius of mismatch ring reduces as time developed $-c f$. Figs. 4(a)4(c)). Finally, the mismatch disappears - Fig. 4(d).

Fig. 5 demonstrates energy dissipation in both cases. It agrees with the analysis in Section 3. System with hydrodynamic effects admits easier development into homogeneous nematic state.

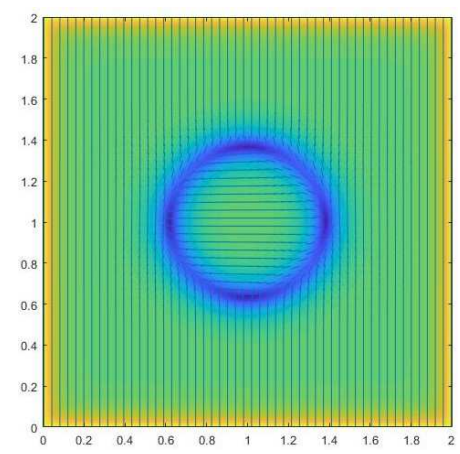

a)

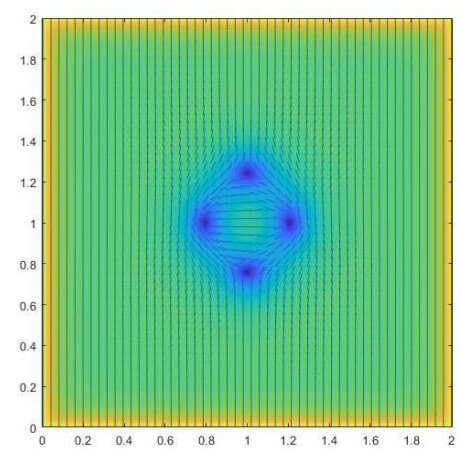

c)



b)

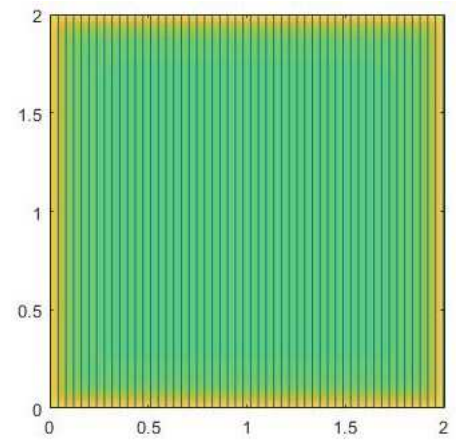

d)

Figure 3: Director and order parameters (in color). Figures (a), (b), (c) and (d) respectively correspond $t=10, t=25, t=50$ and $t-100$. 


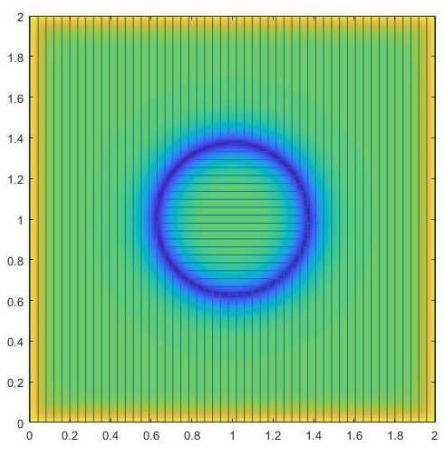

a)

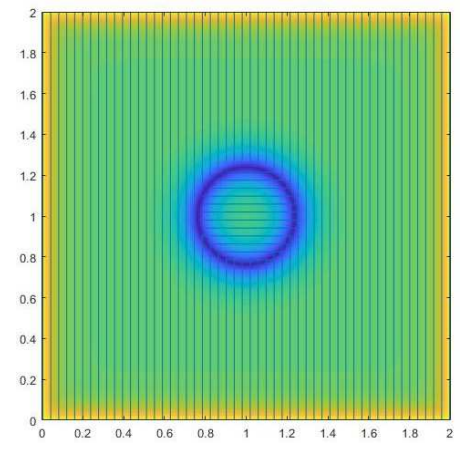

c)

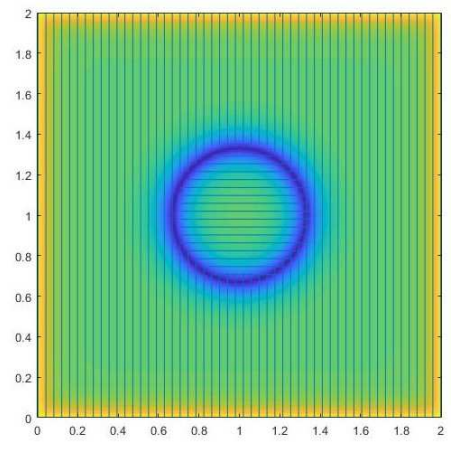

b)

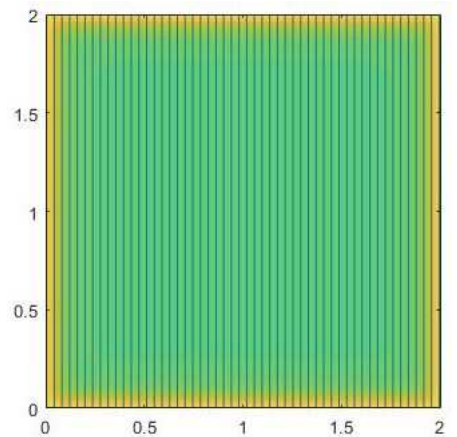

d)

Figure 4: Director and order parameters (in color). Figures (a), (b),(c) and (d) respectively correspond $t=10, t=25, t=50$ and $t-100$.

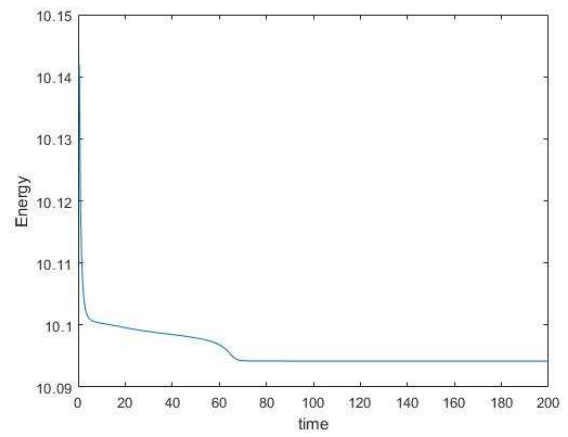

a) under hydrodynamic effect

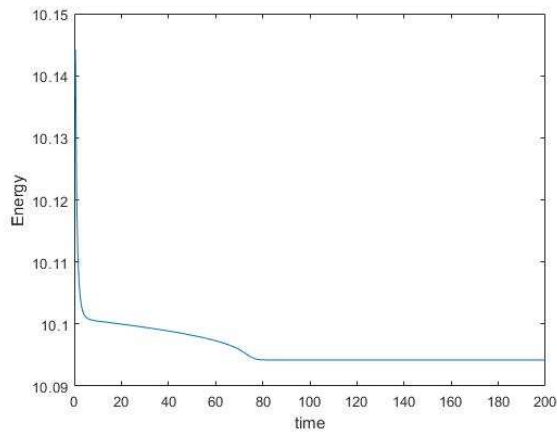

b) without hydrodynamic effect

Figure 5: Total energy versus time under different hydrodynamic effects.

In the last example, we examine the impact of anchoring boundary conditions on the liquid crystal dynamics. The initial conditions are the same as before but the boundary conditions are changed. The computational domain is $\left[0, L_{x}\right] \times\left[0, L_{y}\right], L_{x}=2, L_{y}=1$ and there is an initial defect of +1 type at the point $(1.0,0.5)$. The forces on the $x$ - and $y$-axes exerted on point $(1.0,0.5)$ are different and the initial defect is unstable. The strong 
anchoring boundary condition for $\mathbf{Q}$ in situation considered in Fig. 6 has the form

$$
\left.\mathbf{Q}\right|_{\partial \Omega}=\frac{\tilde{\mathbf{n}}_{0} \tilde{\mathbf{n}}_{0}^{T}}{\left\|\tilde{\mathbf{n}}_{0}\right\|^{2}}-\frac{\mathbf{I}}{2}, \quad \tilde{\mathbf{n}}_{0}=\left(x-0.5 L_{x}, y-0.5 L_{y}\right)^{T} .
$$

The initial defect breaks into two $+1 / 2$ type defects. The $y$-axis boundary is closer to the defect than the $x$-axis and anchoring is strong. Therefore, the break is easier in the $x$-axis

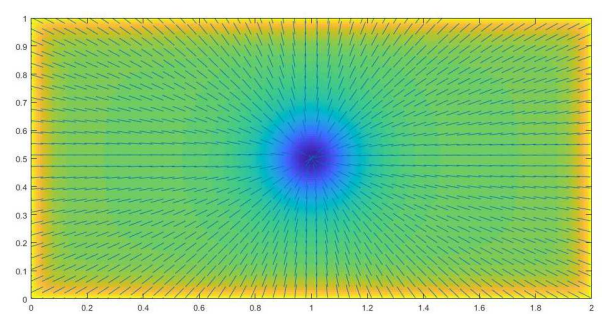

a)

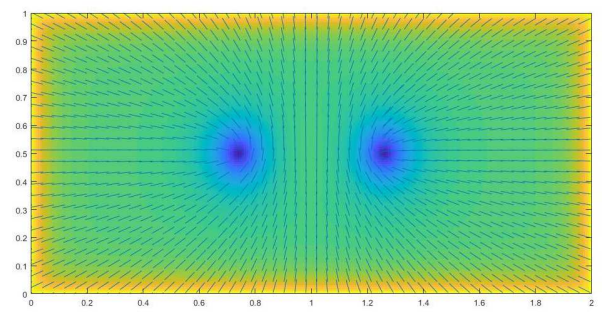

c)

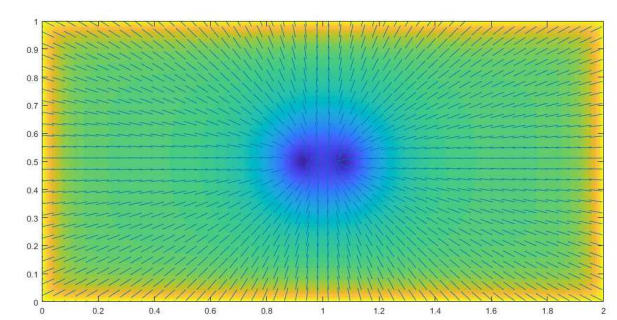

b)



d)

Figure 6: The director and order parameters under strong anchoring, $t=5,50,100,150$.

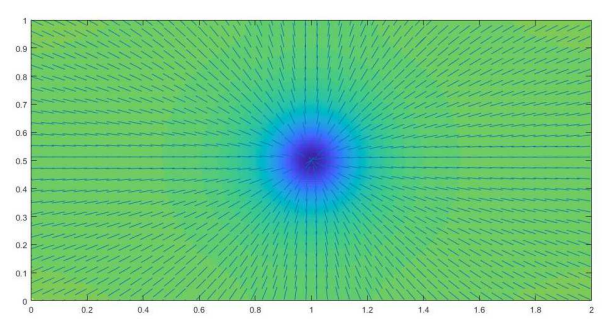

a)

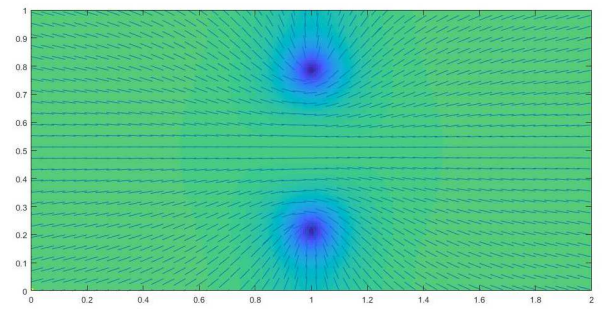

c)

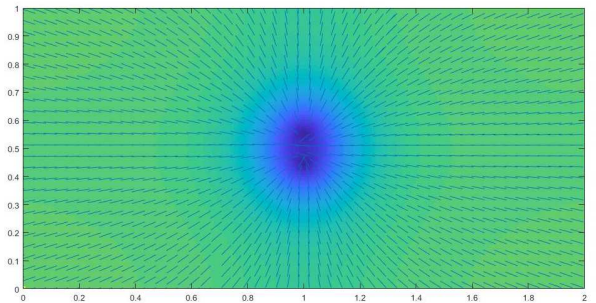

b)

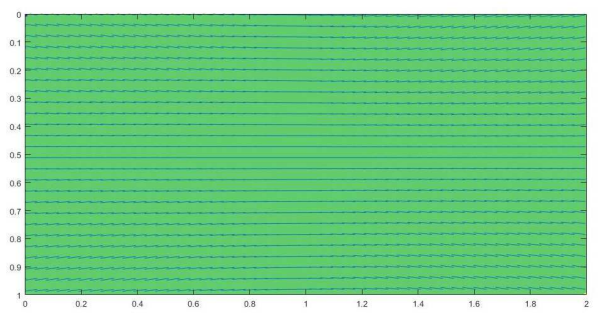

d)

Figure 7: The director and order parameters under weak anchoring, $t=5,50,100,200$. 
direction. In situation considered in Fig. 7, the weak anchoring boundary condition for $\mathbf{Q}$ has the form

$$
\frac{\partial \mathbf{Q}}{\partial \mathbf{n}}=0 .
$$

This defect also breaks up into two $+1 / 2$ type defects. However, because of the weak anchoring on the boundary, the defect breaks easier in the $y$-axis direction. Fig. 8 shows the energy dissipation of the systems under weak and strong anchoring effects. The results are consistent with the analysis of Section 3.

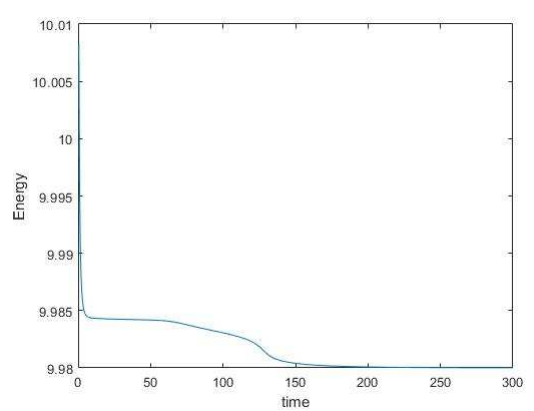

a) Weak anchoring

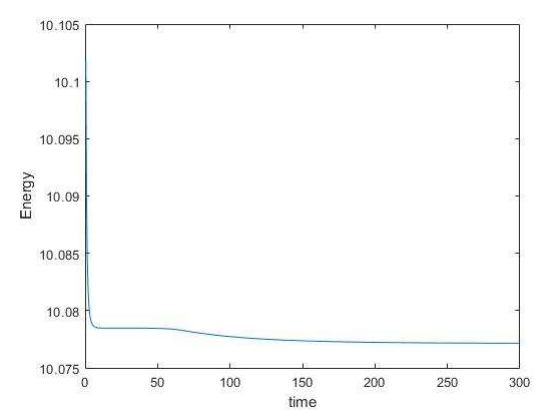

b) Strong anchoring

Figure 8: Energy versus time for different anchoring conditions.

\section{Conclusion}

We construct a BDF2 linear energy-stable numerical scheme for a general tensor-based model of liquid crystals and test it on several examples. Numerical results show the secondorder convergence and energy dissipations, consistent with the theoretical analysis. We also compare the dynamics of the defects with different hydrodynamic effects and anchoring conditions.

\section{Acknowledgments}

This work is partially supported by the National Natural Science Foundation of China (Grant Nos. 11571045, 11671052, 11871105), by the State Scholarship Fund of CSC (Grant No. 201406045016) and by the Fundamental Research Funds for Central Universities (Grant No. 2015KJJCB23).

\section{References}

[1] A.N. Beris and B. Edward, Thermodynamics of flowing systems, Oxford Science Publications (1994).

[2] C. Denniston, E. Orlandini and J.M. Yeomans, Lattice Boltzmann simulations of liquid crystal hydrodynamics, Phys. Rev. E 63(5), 056702 (2001). 
[3] A.E. Diegel and S.W. Walker, A Finite element method for a phase field model of nematic liquid crystal droplets, Commun. Comput. Phys. 25, 155-188 (2019).

[4] J. Fan and T. Ozawa, Regularity criteria for a coupled Navier-Stokes and Q-tensor system, International Journal of Analysis 718173 (2013).

[5] M.G. Forest and Q. Wang, Hydrodynamic theories for mixtures of polymers and rodlike liquid crystaline polymers, Phys. Rev. E 72, 041805 (2005).

[6] M.G. Forest, Q. Wang and H. Zhou, Homogeneous biaxial patterns and director instabilities of liquid crystal polymers in axial and planar elongation, Physics of Fluids 12, $490-498$ (2000).

[7] P.G. de Gennes and J. Prost, The physics of liquid crystals, Oxford University Press (1993).

[8] F. Guillen-Gonzalez and M.A. Rodriguez-Bellido, Weak time regularity and uniqueness for a Qtensor model, SIAM J. Math. Anal. 46(5), 3540-3567 (2014).

[9] F. Guillen-Gonzalez and M.A. Rodriguez-Bellido, Weak solutions for an initial boundary Qtensor problem related to liquid crystals, Nonlinear Anal. 112, 84-104 (2015).

[10] F.M. Leslie, The theory of flow phenomena in liquid crystals, Advances in Liquid Crystals 4, 1-81 (1979).

[11] C.S. MacDonald, J.A. Mackenzie and A. Ramage, Efficient moving mesh method for q-tensor models of nematic liquid crystals, SIAM J. Sci. Comput. 37(2), 215-238 (2015).

[12] D. Marenduzzo, E. Orlandini and J.M. Yeomans, Hydrodynamics and rheology of active liquid crystals a numerical investigation, Phys. Rev. Lett. 98, 118102 (2007).

[13] M. Paicu and A. Zarnescu, Energy dissipation and regularity for a coupled Navier-Stokes and Q-tensor system, Arch. Ration. Mech. Anal. 203, 45-67 (2012).

[14] A. Ramage and A.M. Sonnet, Computational fluid dynamics for nematic liquid crystals, BIT (2015).

[15] J. Shen, J. Xu and J. Yang, The scalar auxiliary (SAV) approach for gradient flows, J. Comput. Phys. 353(15), 407-416 (2018).

[16] J. Shen, J. Xu and J. Yang, A new class of efficient and robust energy stable schemes for gradient flows, SIAM Rev. 61(3), 474-506 (2019).

[17] A.M. Sonnet, P.L. Maffettone and E.G. Virga, Continuum theory for nematic liquid crystals with tensorial order, J. Non-Newton. Fluid Mech. 119, 51-59 (2004).

[18] Géza Tóth, Colin Denniston and J.M. Yeomans, Hydrodynamics of domain growth in nematic liquid crystals, Phys. Rev. E 67, 051705 (2003).

[19] T. Tsuji and A.D. Rey, Effect of long range order on sheared liquid crystalline materials, part I: compatability between tumbiling and behavior and fixed anchoring, J. Non-Newton. Fluid Mech. 73, 127-152 (1997).

[20] Q. Wang, A hydrodynamic theory of nematic liquid crystalline polymers of different configurations, The Journal of Chemical Physics. 116, 9120-9136 (2002).

[21] Y. Yan, W.B. Chen, C. Wang and S.M. Wise, A sencond-order energy stable BDF2 numerical scheme for the Cahn-Hilliard equation, Commun. Comput. Phys. 23, 572-602 (2018).

[22] X. Yang, M.G. Forest, W. Mullins and Q. Wang, 2-D lid-driven cavity flow of nematic polymers: an unsteady sea of defects, Soft Matter 6, 1138-1156 (2010).

[23] J. Zhao and Q. Wang, Semi-discrete energy-stable schemes for a tensor-based hydrodynamic model of nematic liquid crystal flows, J. Sci. Comput. 68, 1241-1266 (2016).

[24] J. Zhao, X.F. Yang, Y.Z. Gong and Q. Wang, A novel linear second order unconditionally energy stable scheme for a hydrodynamic Q-tensor model of liquid crystals, Comput. Methods Appl. Mech. Engrg. 318(1), 803-825 (2017). 